\title{
An origami-inspired cargo drone
}

\author{
P.M. Kornatowski, S. Mintchev, Member, IEEE, and D. Floreano, Senior Member, IEEE*
}

\begin{abstract}
Multicopters stand to revolutionize parcel delivery because of their capability to operate in areas with unsuitable road infrastructure and precisely maneuver in cluttered environments. However, current multicopters for delivery can be dangerous for people, and are difficult to store and transport. Safety issues arise because users are exposed to unshielded spinning propellers. Transportation to the place of deployment and storage is often impaired by the large size that is required for heavy lifting. This paper addresses these limitations by proposing the integration of a quadcopter into a foldable protective cage. The cage provides an all-round protective structure that physically separates the propellers from the environment, ensuring the safety of people. The drone and the cage can be easily folded with a single movement, significantly reducing its size for ease of storage and transportation. This design has been validated with a quadcopter that can lift parcels up to $500 \mathrm{~g}$ and reduce its storage volume by $92 \%$ when folded.
\end{abstract}

\section{INTRODUCTION}

DRONES are rapidly becoming a cost and time effective solution to deliver parcels in densely populated environments as well as in remote locations without a suitable road network [1]. Their capability to navigate above obstacles along the shortest route is capturing attention from companies seeking affordable solutions for cargo transportation and delivery. Most cargo drone prototypes are multicopters [2-4] that leverage vertical take-off and landing capabilities to deliver parcels precisely, even in cluttered environments. Furthermore, the growing popularity of drones for consumers could pave the way to new e-commerce models where drones could enable peer-to-peer transportation of goods. Both scenarios require hovering platforms that are intrinsically safe and easy to store and transport, requirements that are not yet fulfilled by commercially available platforms.

Concerning safety, unshielded spinning propellers are a serious threat and can cause injuries or damages when interacting with people or obstacles. Commercially available drones for delivery provide only limited protection. Lightweight hulls (such as the Parrot AR.Drone 2.0) or small plastic elements around the drone (such as the DJI Phantom 4) only protect the propellers from side contact with objects and are not very effective for users' safety. A safer approach is to enclose the drone into all-round protective structures. For example, lightweight carbon fiber cages have shown to be effective in avoiding injuries to users and avoiding drone damage during collisions [5-8]. However, none of these platforms are directly suited for parcel transportation due to the limited space inside the cage. Moreover, those cages are

*The authors are with the Laboratory of Intelligent Systems (http://lis.epfl.ch) at Ecole Polytechnique Fédérale de Lausanne (EPFL), CH1015 Lausanne, Switzerland (email: Przemyslaw.kornatowski@epfl.ch). cumbersome structures and lack the ability to be folded for ease of storage and transportation.

Concerning size, transportation of heavy payloads requires large aerial surface in order to generate sufficient lift. This means that the drone is much larger than the parcel and thus is difficult to store, handle, and possibly transport to the deployment location. This is even more problematic when the drone is equipped with all-round protective structures, which further increase its size [7-8]. A possible solution lies in foldable drones that can be large enough to carry a useful payload when fully deployed, while being transportable when folded and stowed [9]. Although several commercial foldable hovering platforms are available, such as, DJI Spreading Wings S1000plus, Simtoo Dragonfly, none of them are equipped with all-round protective structures. Indeed, according to our knowledge there are not foldable drones equipped with integrated all-around protective structures that can be simultaneously folded with the drone.

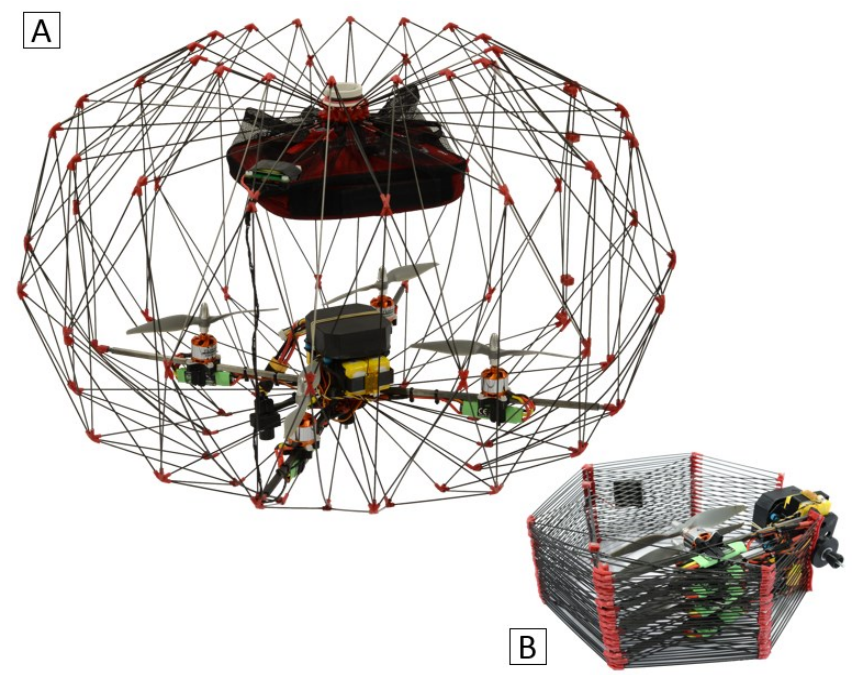

Figure 1. A safe and foldable quadcopter for cargo delivery. (A) Deployed configuration with an enclosed first-aid kit held by a net. (B) Folded configuration with a volume reduction of $92 \%$.

Here we describe a novel design that addresses both the safety and size issues and consists of a foldable protective cage integrated with a multicopter, as presented in Figure 1.

The cage encloses the entire multicopter including the parcel. During flight, the cage is closed to protect the users from the spinning propellers. A safety mechanism shuts down the propellers when the cage is open, reducing the risk of injuries while loading the cargo. The cage is inspired by origami and 
allows the drone to be manually folded with an intuitive operation in order to reduce its volume for ease of storage and transportation. With this new design, a recipient can easily and safely catch the approaching drone. During commercial delivery, the all-round cage coupled with the propeller disengagement system protects inexperienced users from harmful injuries while in close proximity to the drone or while loading it. The foldable structure allows users to easily carry the platform and deploy it in seconds when required. For example, the prototype in Figure 1 fits into a backpack when folded.

\section{CONCEPT}

The cage functions as an all-round protective structure that separates the harmful propellers of the multicopter from the outside environment and people. We resort to an origami design to make the drone foldable with a simple arm movement. Origami structures have been shown to achieve high strength-to-weight ratios [10-11] and a significant size reduction by folding [12-13]. Among the possible origami designs [14-15] (Fig. 2A, 2B), the drone cage is inspired by the pattern used for foldable shelters [16] (Fig. 2C). This pattern can be adapted to obtain a foldable cage similar to a Chinese lantern (Figures 2A and B).
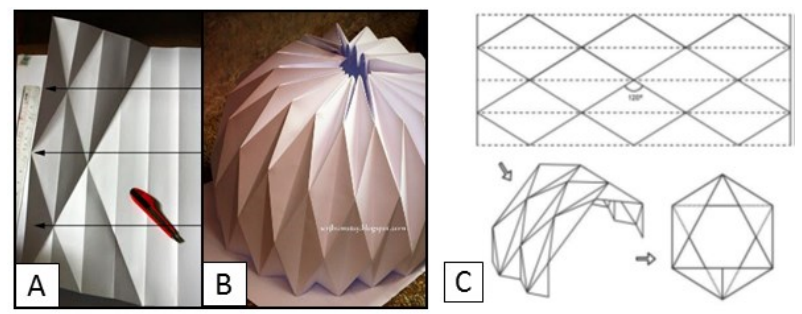

Figure 2. A paper origami lantern [14-15]: (A) the pattern of creases required to create the lantern structure; (B) the deployed structure. (C) The foldable origami-based shelter and its 3 stages of deployment [16].

Traditional origami structures [12] are composed of tiles joined by folds. In the cage, tiles are replaced by struts connected by flexible joints in order to obtain a spatial structure that does not obstruct the airflow generated by the enclosed propellers. The cage has a modular design composed of a repetition of multiple foldable segments (Figure $3 \mathrm{~A}$ ). Each segment is the result of a tessellation of congruent isosceles triangles where the edges are struts and the vertices are flexible joints. The spatial structure of the cage (Figure $3 \mathrm{D}$ ) is obtained by connecting the free ends of multiple segments forming the top and bottom joint. The cage can be folded by pushing apart from each other the first and the last segment (Figure 3F). All the segments fold rotating around the central axis of the cage marked as red dot-dashed line (Figure F). In doing so, the top and bottom central joints get closer to each other, resulting into the final folded polygonal shape of the cage.

The dimensions of the cage in the folded and deployed configurations can be described by a small number of parameters, as illustrated in the flat configuration of the segments (Figure $3 \mathrm{~A}$ ). $L$ is the length of the longer edge of the triangles (struts marked in red color) and corresponds to radius $R$ and height $H$ of the internal empty space in the cage (Figure 3B). Thus, $L$ is also the radius of the footprint of the multicopter. $\beta$ is the apex angle of the triangles and is influenced by the number of segments. The value of $\beta$ defines the distance $h$ between the central top and bottom joints (see Figure 3C). Together, $R$ and $H$, define the internal volume of the cage available for the drone and the cargo. $l$ is the length of the shorter arm of the triangle and its distance is a function of $L$ and $\beta$. In the deployed configuration the angle $\alpha$, between $R$ and $H$, is 90 degrees.
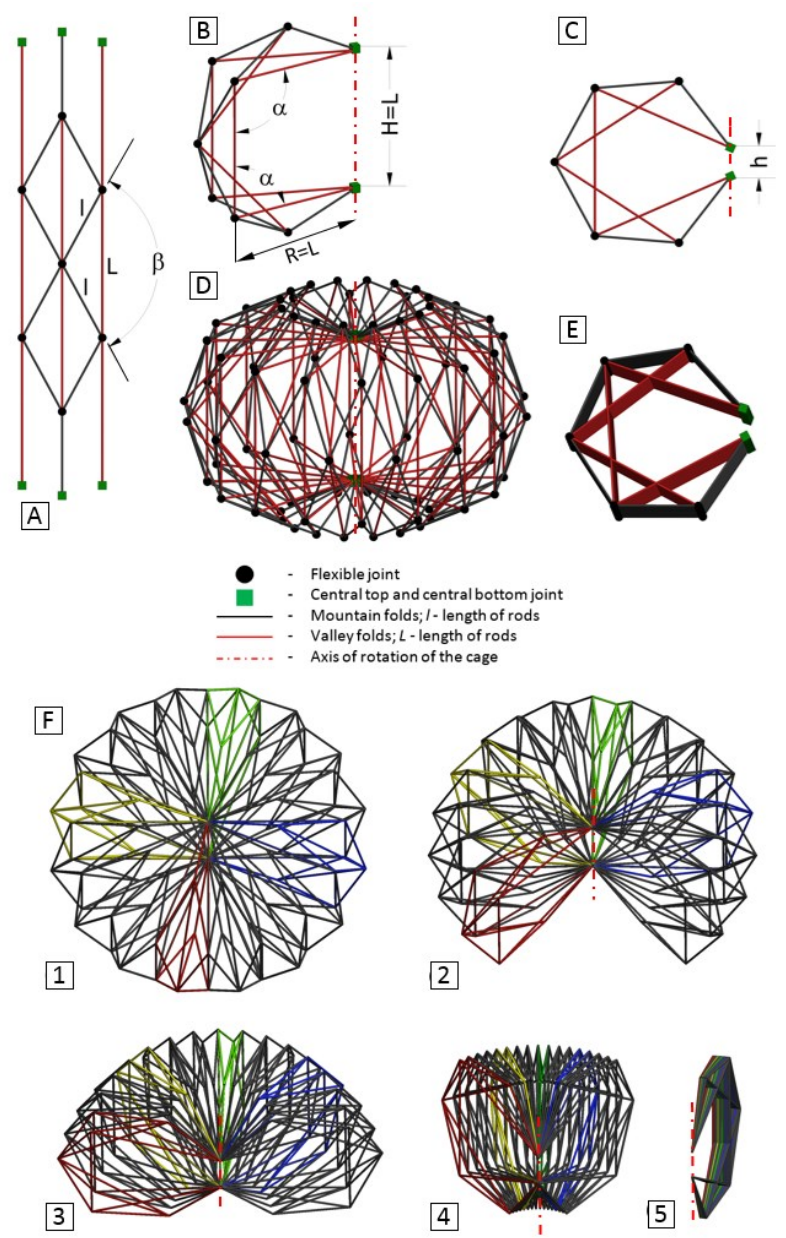

Figure 3. (A) Top view of the flat pattern of one basic segment of the cage before assembling. (B) The basic segment presented in the deployed configuration. (C) Shape of the folded basic segment. Colors represents mountain folds (black) and valley folds (red). (D) Example cage in the fully deployed state consisting of 16 basic segments. (E) Fully folded cage in an isometric view. (F) Folding process of the cage. For sake of clarity every fourth segment is marked with a different color.

The design of the cage presents four important features that make it suitable for cargo delivery. Firstly, the folding pattern allows the cage to be rigid in the deployed configuration, thus ensuring stability during flight. Other origami structures, such as the "magic ball" [17-18], can be squeezed in the deployed configuration. Secondly, the modular structure enables control of the spatial density of the cage. A dense cage with a high number of segments provides more safety at the expense of increased drag, smaller payload, and consequently shorter energetic autonomy of the drone. Thirdly, in the folded 
configuration the cage offers sufficient free space to encapsulate the components of the multicopter and protect them during transportation.

The cage integrates the multicopter and the cargo (Figure 1). The cargo position was chosen above the multicopter in order to avoid obstructing the airflow generated by the propeller, which would result in reduced efficiency (see Section V). That is why the multicopter is integrated in bottom part of the cage. The arms of the multicopter replace some struts of the cage, making the two structures seamlessly integrated. This integration has two advantages: weight reduction as structural components can be shared, and simplified operation as a single arm movement folds both the cage and the multicopter structure.

\section{IMPLEMENTATION}

In order to validate the design presented in the previous section, here we describe the manufacturing of a drone that can carry $0.5 \mathrm{~kg}$ parcels (the typical weight of a first-aid kit). Its total take-off weight (with the parcel) is equal to $1.5 \mathrm{~kg}$. The drone has a size of $65 \times 65 \times 43 \mathrm{~cm}$ when deployed, and folds down to a size of $31 \times 38 \times 12 \mathrm{~cm}$ when stowed.

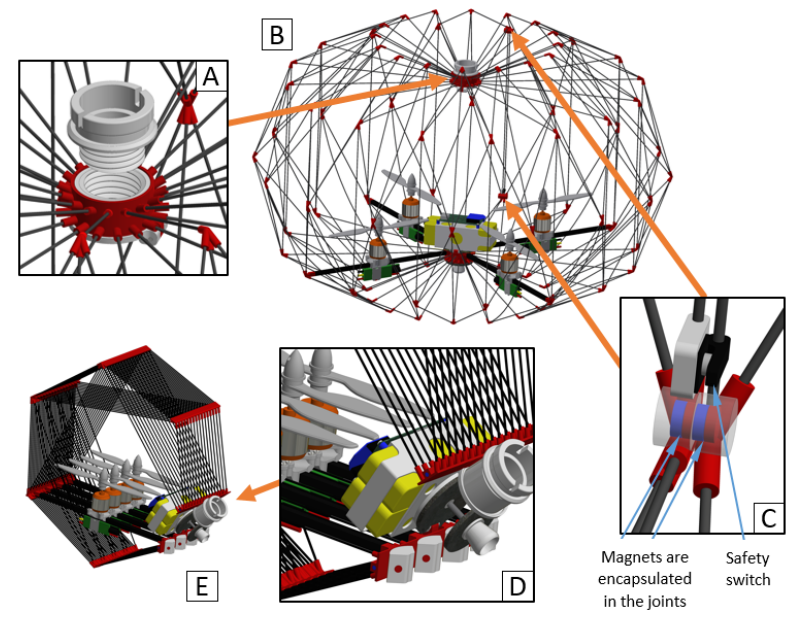

Figure 4. (A) The top locking mechanism. (B) The deployed drone. (C) The side locking mechanism with the safety switch that disable the propulsion system when the cage is open. (D) A zoom-in of the bottom and top locking mechanisms in folded state of the cage. (E) The folded state of the drone.

\section{A. Cage}

The cage is manufactured with carbon-fiber tubes connected by soft joints that are $3 \mathrm{D}$ printed using a flexible material (NinjaFlex ${ }^{\circledR}$ Flexible 3D Printing Filament). Since the cage can be manufactured flat, the joints can be also made using an overmolding technique by locally injecting soft polymers over the tubes. Compared to conventional foldable structures composed of rigid hinged joints, the flexible joints provide smooth folding without affecting the cage rigidity when deployed, which is ensured by the strength of the carbon-fibers tubes. According to the calculations presented in the Appendix, a $0,5 \mathrm{~kg}$ parcel can be hung from the top joint of the cage and cause only minor deformations (see Section V) when $1.5 \mathrm{~mm}$ carbon tubes are used (wall thickness $0.5 \mathrm{~mm}$ ). The rigidity of the cage is mandatory to prevent undesired oscillations of the cargo during flight that could destabilize the drone.

As mentioned earlier, the cage can be made of a variable number of segments. The prototype described here is composed of 16 segments, a number that provides enough protection for users (openings in the cage are smaller than a fist of an adult man), without significant loss of aerodynamic thrust (Section V).

The central top and bottom joints of the cage are composed of a 3D printed flexible strip and multiple connections for the tubes. This part takes the shape of a hollowed cylinder when the cage is deployed (Figure 4A) and is flattened when the cage is folded (Figure 4D). This design prevents interference of tubes during folding and allows to achieve a flat configuration of the edges of the cage when stowed (Figure 4E). A locking mechanism that prevents the opening of the cage during flight is integrated in the top and bottom joints. This mechanism is based on a screw system that is manually operated by the user who tightens the joints before take-off. The cage is also equipped with a side locking mechanism that connects the vertical carbon tubes of the first and last segments of the cage. The side locking mechanism consists of two pairs of cylindrical magnets encapsulated in the flexible joints of the cage (see Figure 4C). To open the cage, those segments have to be pushed apart from each other (see opening process in Figure 3F), thus unlocking the mechanism.

\section{B. Multicopter integration}

Four of the bottom horizontal tubes are replaced by pultruded carbon fiber $6 \mathrm{~mm}$ square tubes that hold the motors of the multicopter. These stiffer tubes prevent undesired vibrations and oscillations of the motors that could lead to instability during flight and compromise reactivity and energetic efficiency. Additionally, the arms of the multicopter are merged with the bottom joint of the cage and secured before flight by the locking mechanism of the cage.

The battery and the autopilot are housed in a frame directly connected to the bottom joint of the cage. The autopilot is the PixHawk board with PX4 software framework.

In order to further enhance user safety, the drone is equipped with a mechanism that cuts the power to the propellers as soon as the cage is open for loading/unloading operations. The safety mechanism comprises two switches that are installed next to the side locking mechanism, between the segments of the cage (Figure 4B-C). As soon as the cage is open, they automatically disengage the propulsion system.

\section{Cargo Integration}

The cargo can be manually connected to the top joint of the cage with two alternative interfaces. The first interface is composed of a round shape lightweight net and ropes. The edges of the net are attached with ropes to the top central joint while the object is placed in the center of the net. This method is very versatile allowing objects with different shapes to be rigidly and easily secured. Although, parcels are subjected to swing oscillations when they do not have a shape that allows them to lay close to the central top joint. This problem is solved by the second type of interface - rigid boxes made out 
of card or thin plastic. However, boxes are heavier than a net and many of them are required to deliver objects with different shapes and sizes. These two interfaces are therefore complementary solutions which depend on the transported cargo.

\section{SCALABILITY}

Here we describe a scalability model to calculate the size of the drone given the payload and the flight time. The model takes the desired flight time and cargo mass as input and generates as output, firstly the dimensions and mass of the multicopter and secondly the dimensions of the tubes and mass of the cage (Figure 5). The model consists of three sub models: mass and power model of the quadcopter, geometrical model of the cage, and rigidity model of the cage.

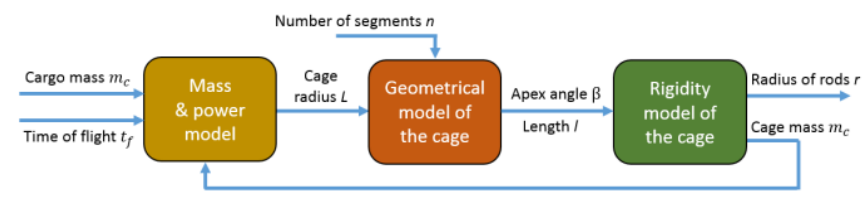

Figure 5. The dimensioning and scalability model of the drone composed of mass and power model, geometrical and rigidity model of the cage.

In the first step, a mass and power model described in [19] (see also Appendix) is used to compute the footprint of the drone for a given payload and time of flight. The resulting footprint is used to compute the length $L$ of the tubes that correspond to the valley folds of the cage. In the second step, a geometrical model of the cage is used to compute the remaining parameters that define the cage geometry $(l$ and $\beta)$. In the third step, a rigidity model computes the minimum radius of the tubes that prevent buckling of the cage under the cargo load, thus ensuring flight stability. The same model also computes the total mass of the cage considering the density of the material of the tubes. The resulting cage mass is fed back to the mass and power model where it is added to the total mass of the drone to compute a new and higher value for the footprint. The entire process is iterated until the difference between the $L$ values of two consecutive iterations is less than $5 \%$.

The model predicts that the radius of the footprint of the multicopter grows slower than the mass of the cargo (Figure 6A). For example, when the cargo mass increases 3 times, the radius of the footprint of the multicopter increases less than 2 times. In parallel, the volume reduction of the folded multicopter decreases with cargo mass (Figure 6B). Both cases reveal that the drone can be well scaled up in terms of size.

The mass of the cage grows linearly with the mass of the cargo (see Figure 6C). The model also predicts that the ratio between the mass of cage to the total mass increases slowly and linearly with the cargo mass (Figure 6D).

The same behavior as presented in Figure $6 \mathrm{D}$ can be observed when the time of flight is changed for given payload. The cage to total mass ratio increases with the time of flight because of the increased dimensions.

These results reveal that the cage can be conveniently scaled up for larger payloads.

\section{EXPERIMENTAL VALIDATION}

\section{A. Verification of the dimensioning model of the drone}

The model predictions match the values measured on the prototype designed for $0.5 \mathrm{~kg}$ cargo and 10 minutes of flight (Table I). In a series of 15 flight tests (hovering on the spot) with $0.5 \mathrm{~kg}$ payload, we measured $10 \pm 1 \%$ minutes of hovering time, which is comparable to the 10 minutes predicted by the model.
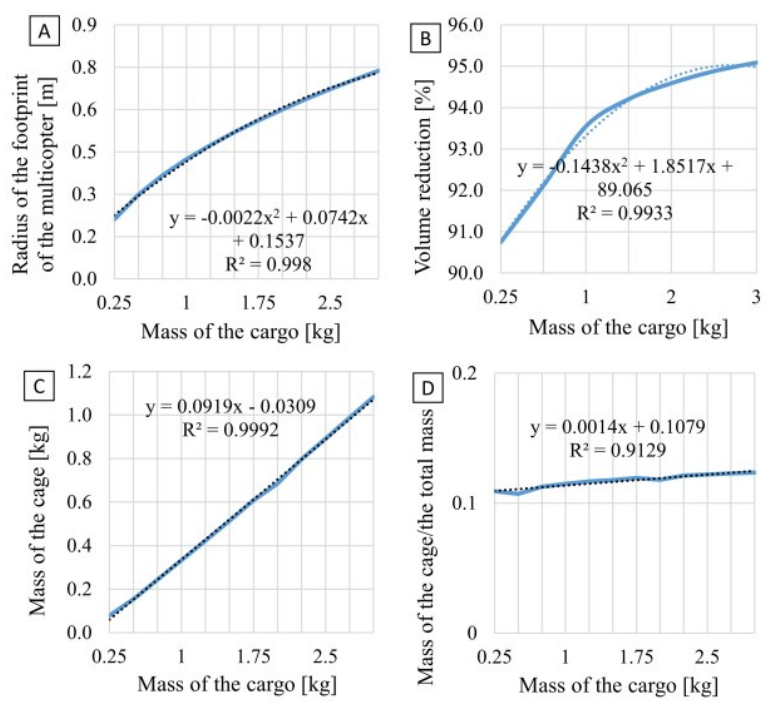

Figure 6. The plots present different parameters of the drone as a function of the mass of the cargo. Parameters: (A) Radius of the footprint of the multicopter. (B) Volume reduction is the ratio between the volume of the deployed and folded drone. (C) The mass of the cage. (D) The ratio between the mass of the cage and the total mass of the drone. The trendlines are shown with a dotted line.

A $10 \%$ difference can be explained by the drag induced by the cage on the airflow generated by the propellers (see next section), which is not considered in the model. Moreover, the test for distance coverage was performed. The drone flew distance of $2 \mathrm{~km}$ with a $0.5 \mathrm{~kg}$ parcel. The distance coverage was tested outdoors with 10 degrees inclination of the multicopter around the pitch axis in the forward flight.

The largest difference between predicted and measured values $(17.4 \%)$ concerns the mass of multicopter (excluding the battery and the cage). However, this difference could be reduced by using different materials or an alternative design for the central bottom locking mechanism, the battery holder or by using more expensive materials, which will be stronger and lighter, such as carbon fiber. Nevertheless, the difference between the predicted and observed total mass is only $6.14 \%$.

TABLE I. MASS COMPARISON BETWEEN PREDICTED AND OBSERVED VALUES OF THE PROTOTYPE

\begin{tabular}{cccc}
\hline \multirow{2}{*}{ Component } & \multicolumn{2}{c}{ Mass [kg] } & Difference \\
\cline { 2 - 4 } & Model & Prototype & $\%$ \\
\hline $\begin{array}{c}\text { Multicopter w/o } \\
\text { battery and cage }\end{array}$ & 0.466 & 0.564 & 17.4 \\
\hline cage & 0.154 & 0.150 & -1.0 \\
\hline battery & 0.316 & 0.319 & 0.94 \\
\hline cargo & 0.5 & 0.5 & 0.0 \\
\hline Total & 1.436 & 1.53 & 6.14 \\
\hline
\end{tabular}


In order to measure the stiffness of the cage under loading, the top central joint (the place of cargo attachment) has been incrementally loaded up to $1.4 \mathrm{~kg}$ while measuring its vertical displacement. The results indicate that the cage remains stiff up to $1.2 \mathrm{~kg}$, but collapses for heavier loads (Figure 8). The vertical displacement is reversible and is due to the buckling of the vertical and oblique top tubes (see yellow dashed lines in Figure 8B). These results indicate that the cage is sufficiently rigid, and thus stable in flight, for the desired cargo load of $0.5 \mathrm{~kg}$.
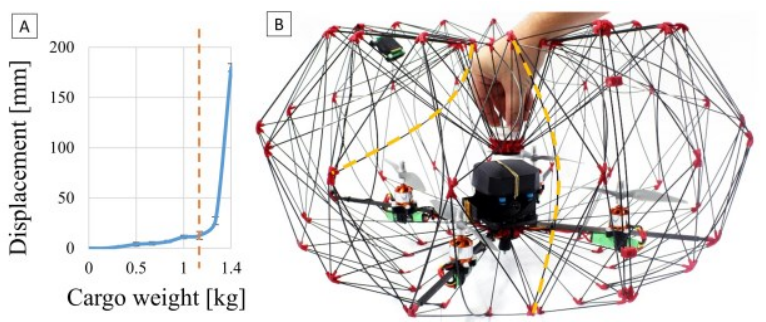

Figure 8. (A) The plot presents experimentally measured displacement of the top central joint of the cage under different cargo loads (continuous blue line). The tubes of the cage start to buckle significantly under the load of $1.2 \mathrm{~kg}$ (dashed red line). Therefore the cage can withstand the desired payload of 0.5 $\mathrm{kg}$ with a safety factor of 2.4. (B) The cage under the load above $1.2 \mathrm{~kg}$ with displaced central top joint. The yellow dashed curves mark two representative buckled tubes.

\section{B. Effect of the cage on drag}

Wind tunnel tests were performed to show how the cage and a parcel (size $28 \mathrm{~cm} \times 28 \mathrm{~cm} \times 6 \mathrm{~cm}$ ) increase the drag of the drone. We tested the drone with four different configurations: (i) unladen drone without the cage, (ii) unladen drone with the cage, (iii) laden drone without the cage, (iv) laden drone with the cage. The propellers were removed for these tests. Additionally, we compared (see Table II) our results with existing values in the literature of similar size drones tested by $[8,20]$.

The drag coefficient is nondimensionalised using the motor to motor area of the drone as a reference. For the comparison with the other results in the literature the equivalent flat plat area (drag coefficient times reference area of the airframe) is also provided. This a value often used in rotary wing vehicle literature.

TABLE II. AVERAGED DRAG COEFFICIENT COMPARISON BETWEEN OUR CAGED DRONE AND OTHER DRONES OF SIMILAR SIZE.

\begin{tabular}{lll}
\hline \multicolumn{1}{c}{ Name } & $\begin{array}{c}\text { Averaged } \\
\text { drag } \\
\text { coefficient }\end{array}$ & $\begin{array}{c}\text { Equivalent } \\
\text { flat plat } \\
\text { area }\left[\mathbf{m}^{2}\right]\end{array}$ \\
\hline Unladen drone without cage & 0.125 & 0.006 \\
\hline Unladen drone with cage & 0.497 & 0.024 \\
\hline Laden drone without cage & 0.723 & 0.035 \\
\hline Laden drone with cage & 1.226 & 0.060 \\
\hline DJI Phantom 3 [20] & 0.326 & 0.020 \\
\hline 3DR Solo [20] & 0.353 & 0.030 \\
\hline 3DR Iris + [20] & 0.271 & 0.029 \\
\hline Straight Up Imaging Endurance [20] & 0.160 & 0.042 \\
\hline Caged drone [8] ${ }^{1}$ & 0.847 & 0.092
\end{tabular}

${ }^{1}$ For the [8] drone the motor to motor dimensions were estimated from the total diameter of the drone.
The results show that the cage increases drag four times compared to the same quadcopter without the cage. However, comparing our caged drone to a DJI Phantom 3 or 3DR Solo (without any protective mechanism around propellers) the average drag coefficient is increased only by one and a half times. This is an acceptable value considering the advantage of the cage in terms of user safety.

The impact of the cage on the drag can be quantified by an averaged difference between the equivalent flat plate areas of the drone with and without the cage, for the both the laden and unladen cases. This is a value of $0.00215 \mathrm{~m}^{2}$, being the average of the difference between the caged and uncaged drone with the package and the difference between the caged and uncaged drone without the package. Taking the average of the difference between the laden and unladen drone with the cage and the laden and unladen drone without the cage gives a value of $0.0325 \mathrm{~m}^{2}$, which is the increase in flat plate area that results from the package. This indicates that the package has a larger effect than the cage. If a similar parcel were to be added to a DJI Phantom 3 or 3DR Solo (without protective mechanisms) they would have similar drag values to the laden drone with the cage.

This allows us to conclude that while the cage has a measurable effect on the drag of the airframe, its impact on the overall aerodynamics is of a similar scale to the impact of a parcel. The overall drag of the airframe with a parcel and the cage is comparable to commercial systems carrying a similar parcel.

\section{Effect of the cage on thrust}

The density of the cage structure can be modified to find an optimal trade-off between safety and flight efficiency. The prototype is composed of 16 vertical segments with an intertube distance of $7.5 \mathrm{~cm}$. This value ensures that a fist of an adult man cannot be horizontally inserted in the cage. Moreover, the number of tubes placed below the propellers cause drag and turbulences that reduce the total thrust generated by the four propellers from $1.2 \mathrm{~kg}$ (without the cage) to $1.06 \mathrm{~kg}$ (with the cage). We find this thrust loss acceptable considering the protection benefits.

\section{Folding procedure}

Before folding the drone, the top and bottom locking joints of the cage have to be unlocked (Figure 4), which takes about 10 seconds.

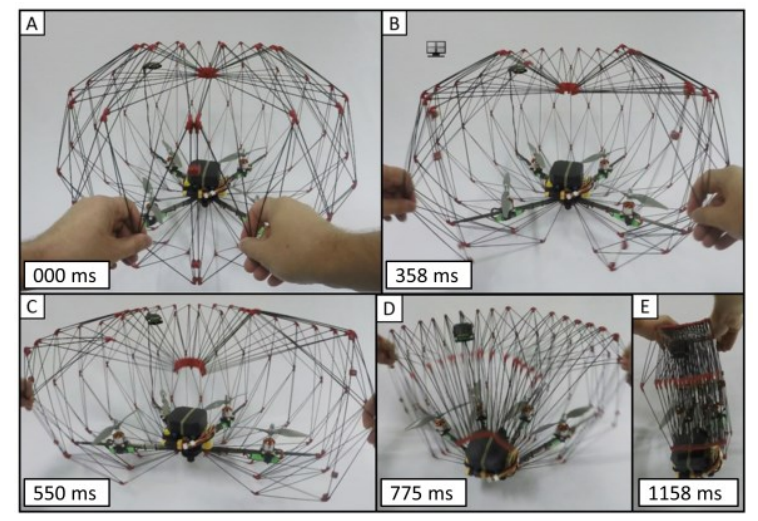

Figure 10. Multiple snapshots from a video captured by a GoPro HERO3 (120 fps) showing unlocking side locking mechanism and the last folding step of the drone. (A) The drone fully deployed. (B) The drone $25 \%$ folded. (C) The drone $50 \%$ folded. (D) The drone $75 \%$ folded. (E) The drone fully folded. 
Afterwards, the folding process is illustrated in Figure 10 and showed in the attached video. First, the user has to unlock the side locking mechanism while opening the cage. Two adjacent segments of the cage have to be pushed away from each other to fold the cage and integrated quadcopter. The folding procedure takes $1.2 \mathrm{~s}$. The deployment process takes the same amount of time and requires the same steps but in reverse order.

Table III presents a comparison of the dimensions of the drone in deployed and folded configurations along with those of a deployed airframe of the foldable quadcopter without the cage. The values given in the table are the dimensions of a cuboid box containing the drone. The cuboid shape approximates the encumbrance of the drone during transportation or while stored on a shelf. Thus, the foldable drone can reduce its storage volume by $92 \%$. The deployed quadcopter without the cage has only a $14 \%$ smaller volume than the folded configuration with the cage.

TABLE III. SIZE COMPARISON BETWEEN FOLDED AND DEPLOYED DRONE WITH THE CAGE, AND NOT FOLDABLE QUADCOPTER WITHOUT THE CAGE

\begin{tabular}{ccccc}
\hline & $\begin{array}{c}\text { Size } \\
{[\mathbf{m}]}\end{array}$ & $\begin{array}{c}\text { Footprint } \\
{\left[\mathbf{m}^{2}\right]}\end{array}$ & $\begin{array}{c}\text { Volume } \\
{\left[\mathbf{m}^{3}\right]}\end{array}$ & $\begin{array}{c}\text { Weight } \\
{[\mathbf{g}]}\end{array}$ \\
\hline $\begin{array}{c}\text { Deployed } \\
\text { configuration } \\
\text { with the cage }\end{array}$ & $0.65 \times 0.65 \times 0.43$ & 0,423 & 0,182 & \\
\cline { 1 - 2 } $\begin{array}{c}\text { Folded } \\
\text { configuration } \\
\text { with the cage }\end{array}$ & $0.31 \times 0.38 \times 0.12$ & 0,118 & 0,014 & \\
\hline $\begin{array}{c}\text { Deployed } \\
\text { configuration } \\
\text { w/o the cage }\end{array}$ & $0.35 \times 0.35 \times 0.1$ & 0,123 & 0,012 & 640 \\
\hline
\end{tabular}

\section{E. In-hand landing}

The rounded protective cage is not only safe for regular handling of the cargo drone, but is also useful in emergency situations where there is no landing spot (Figure 10). Existing cargo drones [21] may deliver the cargo with a tether in these situations. However, a tether may become entangled in obstacles or the recipient of the cargo may pull the tether too hard and cause the drone to destabilize and fall. The proposed foldable drone instead can be safely grabbed as it approaches the recipient (Figure 11) (see attached video).

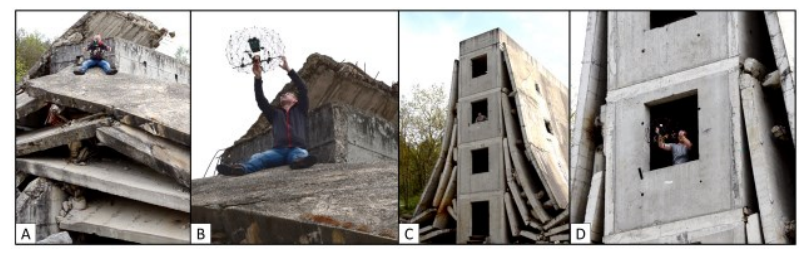

Figure 11. Situations where it is hard to land for a standard drone and where the caged cargo drone can be easily grabbed by a human. (A-B) Person trapped on an uneven terrain of collapsed building. (C-D) Person stuck on a floor of a high building.

\section{CONCLUSION}

We have developed a safe foldable drone for cargo transportation. The quadcopter is equipped with an all around cage that protects people and the drone. The drone can be manually folded for ease of storage and transportation. Moreover, in the folded state, the electromechanical components of the quadcopter are protected inside the structure of the folded cage. To ensure safety for people while removing a parcel from the cage, the drone is equipped with security switches that disengage the propulsion system while the cage is open. The wind tunnel tests revealed that the overall drag of the airframe with a parcel and the cage is comparable to unshielded commercial drones carrying a similar parcel. Model-based predictions on the dimensions of the drone match a physical prototype and suggest that the proposed design could scale up to fly $2 \mathrm{~kg}$ cargo over $15 \mathrm{~km}$, which would cover $86 \%$ of the deliveries made by Amazon.com, Inc. [22].

Future work will investigate other shapes of the cage, adapted for special parcels, such as documents. Delivery of flat documents will allow us to reduce the height of the drone, thus the length of the vertical and oblique tubes in the cage. This will reduce to the weight and drag of the cage and increase the time of flight. To protect the drone from damages caused by falls from high altitudes, a parachute will be installed to its top central joint outside the cage. In order to verify recipient of the package or allow drone to precisely land, additional sensors, such as cameras will be installed on the cage. To facilitate the usage of the drone for less experienced users, the unlocking mechanisms will be redesigned to easier access the cargo placed inside the drone and to faster fold and deploy the cage. Our approach with a foldable protective cage has the potential to increase the cargo deliveries to people. Furthermore, we believe that our solution will revolutionize person to person transportation using drones.

\section{APPENDIX}

\section{A. Mass and power model}

The first step to design the cage is to define the footprint of the multicopter for given time of flight and mass of the transported cargo. To do that and to discuss scalability of the cage, the mass and power model developed in [19] is used. It is transformed to calculate a propeller diameter of the multicopter:

$$
D=\frac{43.45 t_{f} \sqrt{m_{c}}}{R_{b} e_{d} F M \sqrt{\delta n \pi R_{c}}}
$$

where $t_{f}$ is the time of flight, $m_{c}$ is mass of the cargo, $R_{b}$ is the ratio between mass of the battery and a take-off weight, $e_{d}$ is energy density, $F M$ is a figure of merit, $\delta$ is air density, $n$ is number of propellers, $R_{c}$ is the ratio between the mass of the cargo and take-off weight.

Given the radius of the propellers, the radius of the footprint of the quadcopter can be calculated from aerodyanmic considerations. As discussed in [24] the space around the propellers should be around $\sqrt{2}$ times the propeller's radius in order to avoid vortex interpehrences between the propellers. Moreover, additional clearance ( $10 \%$ of the diameter) arround the perimeter of the footprint of multicopter was allowed to provide safety for human fingers during in-hand landing.Thus, the radius of the quadcopter is calculated:

$$
R_{\text {quadcopter }}=1.59 \mathrm{D}
$$


The values of the mass ratios in [19] adopted for high-payload capabilities are as follows: $R_{b}=22 \%, R_{c}=50 \%, R_{s}=28 \%$ (ratio between the mass of the structure and the total take-off mass). We kept the ratio $R_{b}=22 \%$ and changed the two other ratios taking into account the additional mass of the cargo. The remaining value is equally divided, thus $R_{c}$ and $R_{s}$ is equal to $39 \%$. After each iteration, the added mass of the cage to the total take-off mass changes ratios $R_{c}$ and $R_{s}$. Values of parameters assumed in the model: $(F M)-0.333$ (measured for the motor and propeller used in the prototype); the time of flight $\left(t_{f}\right)-10$ minutes; the battery energy density $\left(e_{d}\right)-$ $162.5 \mathrm{Wh} / \mathrm{kg}$; the air density $(\delta)-1.2 \mathrm{~kg} / \mathrm{m}^{3}$; the number of propellers $(n)-4$.

\section{B. Geometrical model of the cage}

After calculating the radius of the footprint of the multicopter which is equal to dimension $L$ of the cage, the number of segments has to be chosen and other dimensions of the cage can be calculated: the apex angle $\beta$, and the shorter arm of the triangle $l$.

As stated before, the number of segments of the cage has to be divisible by the number of arms of the multicopter. There is a relation that has to be respected in order to design the foldable cage. It is due to the fact that $\beta$ has to fit within a certain range. Details of these relations are presented below. In the folded state of the cage, free space between the central top and bottom joints, the dimension $h$ (see Figure 3C) is left on purpose as a place for components of the central part of the robot (autopilot, battery, etc.):

$$
h=L\left(1-2 \cos \left(2 \beta-180^{\circ}\right)\right.
$$

Eq. 3 allows us to conclude that $\beta$ has to be bigger than $120^{\circ}$ to leave space for components. As was presented in section III, the arms of the quadcopter are integrated directly with the structure of the cage. To facilitate this integration, the vertical and horizontal $L$ tubes presented in figure $3 \mathrm{~B}$ have to be perpendicular $\left(\alpha=90^{\circ}\right)$. To keep this position of tubes $\beta$ cannot be greater than $135^{\circ}$.

It is important to remember that $\beta$ changes with the chosen number of segments of the cage, and thus, $h$ can be changed only by changing $L$ for a given number of segments. Table II presents values of apex angles $\beta$ for different numbers of segments. The values which are crossed out do not fit in the range discussed above.

$l$ is the length of the shorter arm of the triangle (rod marked with black color in Figure 3A) and it is dependent on the chosen $L$ and $\beta$ angle:

$$
\begin{aligned}
& l=\frac{L}{2 \sin \left(\frac{\beta}{2}\right)} \\
& \text { C. Rigidity model of the cage }
\end{aligned}
$$

To calculate the mass and the volume of the cage, the radius of the tubes of the cage has to be found. The goal is to find the lightest tubes that will not buckle under the load of the cargo, thus will keep the cargo in a stable position during the flight. To do this, the outer radius of the tube knowing its material properties has to be found. We assumed constant wall thickness of the tube $0.5 \mathrm{~mm}$ in the model (commercially available). The cargo attached to the top central joint of the cage is kept in position due to the rigidity of the tubes $\mathrm{AC}$, marked in red in the single segment (see Figure 12). To emphasize the importance of this tube for the rigidity of the cage, we assume that this tube is removed. Thus, the pyramid EFDC in the top part of the cage would just freely rotate around axis joining the points $\mathrm{E}$ and $\mathrm{F}$. This means that the load from the cargo will be applied mainly to the tube AC. Finding the radius of the tube $\mathrm{AC}$ is therefore crucial to determine the rigidity of the cage with the cargo attached to the central top joint.

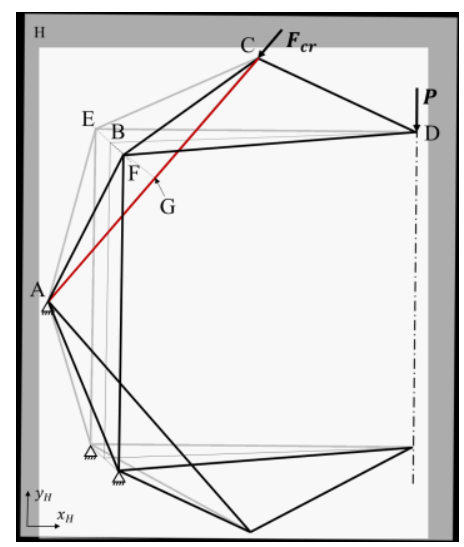

Figure 12. The basic segment of the cage. The view with the distribution of forces on the plain $H$. The visible rod are in front of the plain.

To simplify the design, the calculated radius $r$ for the tube $\mathrm{AC}$ is used also for the other tubes in the cage. Knowing $r$, the mass and the volume of the cage in the folded state can be calculated. A tube under vertical load starts to buckle after reaching a certain force called the critical force $F_{c r}$. To calculate this force for tubes pinned on both sides, standard beam theory is used [24]:

$$
F_{c r}=\frac{\pi^{2} E\left(\frac{\pi\left(R^{4}-r^{4}\right.}{4}\right)}{L^{2}}
$$

where $\mathrm{E}$ is the Young's modulus of the material, $\frac{\pi\left(R^{4}-r^{4}\right)}{4}$ is the tube's area moment of inertia ( $\mathrm{R}$ is outer radius, $\mathrm{r}$ is inner radius), $\mathrm{L}$ is the length of the tube.

The critical force $\mathrm{F}_{\mathrm{cr}}$ acting along the rod $\mathrm{AC}$ under the desired load of the cargo should be calculated. To simplify calculations, the geometry of a single segment is presented in 2D (see Figure 12).

To calculate $\mathrm{F}_{\mathrm{cr}}$ a simple equilibrium of forces is used:

$$
F_{c r} \cdot B G=P \cdot B D
$$

where $\mathrm{BG}$ and $\mathrm{BD}$ are distances from forces $F_{c r}$ and $P$ to rotation point $\mathrm{B}$. The assumption is that the point $\mathrm{B}$ is fixed. $P$ is the force acting on the basic segment of the cage, coming from the weight of the cargo:

$$
P=\frac{s_{f} m_{c} g}{n_{s}}
$$

where $m_{c}$ is the mass of the cargo, $n_{s}$ is the number of segments, $g$ is the gravitational acceleration. The rod starts to buckle just after exceeding the critical force. Therefore, to ensure rigidity of the cage during flight maneuvers with the required cargo on board, a safety factor $s_{f}$ is applied.

Distances $B G$ and $B D$ are found from geometrical relationships in the basic segment of the cage (see Figure 6): 


$$
\begin{aligned}
& B D=L \cos \left(\frac{\pi}{n_{s}}\right) \\
& B G=\sqrt{\left(\frac{L}{2 \sin \left(\frac{\beta}{2}\right)}\right)^{2}-\left(L \sin \left(\frac{\pi}{n_{s}}\right)\right)^{2}-\left(\frac{L}{2}\right)^{2}}
\end{aligned}
$$

Now $r$ can be computed by combining above presented equations.

Knowing $r$, the mass of the cage composed of tubes and joints is calculated from:

$M_{n_{s}}=n_{s} \varrho \pi r^{2}(10 l+5 L)+\left(5 n_{s} m_{\text {joint }}\right)$

where $\varrho$ is density of the material of the rod and $m_{\text {joint }}$ is the mass of one joint.

\section{ACKNOWLEDGMENT}

This work was supported by the Swiss National Science Foundation through the National Centre of Competence in Research Robotics. The authors would like to gratefully acknowledge Gregorie Marie Heitz for his support to the project: adapting the autopilot to this specific drone to allow it to be controlled in manual and autonomous mode; as well as for being a pilot during the tests. Additionally we would like to acknowledge Cameron Dowd for his support with performing a wind tunnel tests of our drone.

\section{REFERENCES}

[1] D. Floreano and R. J. Wood, "Science, technology and the future of small autonomous drones," Nature, vol. 521, no. 7553, pp. 460-466, 2015.

[2] D. Bamburry, "Drones: Designed for Product Delivery," Des. Manag. Rev., vol. 26, no. 1, pp. 40-48, 2015. R. D'Andrea, "Guest editorial can drones deliver?" IEEE Transactions on Automation Science and Engineering, vol. 11, no. 3, pp. 647-648, 2014.

[3] Interspot. Robots Will Soon Deliver Both Medicine And Mail. [Online]. Retrieved from http://inventorspot.com/articles/robots_will_soon_deliver_both_medi cine and mail, 2 September 2015.

[4] The Economic Times. "Switzerland begins postal delivery by drone," [Online]. Retrieved from http://economictimes.indiatimes.com/slideshows/nationworld/switzerland-begins-postal-delivery-bydrone/slideshow/47998181.cms, 2 September 2015.

[5] A. Klaptocz, A. Briod, L. Daler, J. C. Zufferey, and D. Floreano, "Euler spring collision protection for flying robots," in IEEE International Conference on Intelligent Robots and Systems, 2013, pp. $1886-1892$.

[6] A. Kalantari and M. Spenko, "Design and experimental validation of HyTAQ, a Hybrid Terrestrial and Aerial Quadrotor," in Proceedings IEEE International Conference on Robotics and Automation, 2013, pp. $4445-4450$.
[7] A. Briod, P. Kornatowski, J. C. Zufferey, and D. Floreano, "A collision-resilient flying Robot," J. F. Robot., vol. 31, no. 4, pp. 496$509,2014$.

[8] C. J. Salaan, Y. Okada, K. Hozumi, K. Ohno, and S. Tadokoro, "Improvement of UAV's flight performance by reducing the drag force of spherical shell," in IEEE International Conference on Intelligent Robots and Systems, vol. 2016-November, pp. 1708-171, 2016.

[9] S. Mintchev, L. Daler, G. L'Eplattenier, L. Saint-Raymond, and D. Floreano, "Foldable and self-deployable pocket sized quadrotor," in 2015 IEEE International Conference on Robotics and Automation (ICRA), 2015, pp. 2190-2195.

[10] S. S. Tolman, I. L. Delimont, L. L. Howell, and D. T. Fullwood, "Material selection for elastic energy absorption in origami-inspired compliant corrugations," Smart Mater. Struct., vol. 23, no. 9, p. 94010, 2014

[11] M. Schenk and S. D. Guest, "Origami folding: A structural engineering approach,” Origami 5 Fifth Int. Meet. Origami Sci. Math. Educ., pp. 1-16, 2011.

[12] E. A. Peraza-Hernandez, D. J. Hartl, R. J. Malak Jr, and D. C. Lagoudas, "Origami-inspired active structures: a synthesis and review," Smart Mater. Struct., vol. 23, no. 9, p. 94001, 2014.

[13] L. Dufour, K. Owen, S. Mintchev, and D. Floreano, "A drone with insect-inspired folding wings," in IEEE International Conference on Intelligent Robots and Systems, 2016, vol. 2016-November, pp. $1576-1581$.

[14] R. J. Lang, Origami 4, vol. 10, 2009.

[15] Serjbumatay Blogspot. Retrieved from http://serjbumatay.blogspot.ch/2011/04/how-to-make-origami-paperlantern.html, 2 August 2016.

[16] De Temmerman, I.A.N., Mollaert, M., Van Mele, I.A.T. and De Laet, I.A.L., 2007. "Design and analysis of a foldable mobile shelter system." International Journal of Space Structures, 22(3), pp.161-168.

[17] D.-Y. Lee, J.-S. Kim, S.-R. Kim, J.-S. Koh, and K. Cho, "The deformable wheel robot using magic-ball origami structure," Proc. ASME 2013 Int. Des. Eng. Tech. Conf. Comput. Inf. Eng. Conf. IDETC/CIE 2013, pp. 1-9, 2013

[18] Le, P.H., Molina, J. and Hirai, S., "Application of Japanese Origami Ball for Floating Multirotor Aerial Robot," World Academy of Science, Engineering and Technology, International Journal of Mechanical, Aerospace, Industrial, Mechatronic and Manufacturing Engineering, 8(10), pp.1740-1743, 2014.

[19] Roberts, J., "Enabling Collective Operation of Indoor Flying Robots,"PhD Thesis, Ecole Polytechnique Federale de Lausanne, 2011.

[20] Russell, C.R., Jung, J., Willink, G. and Glasner, B., "Wind Tunnel and Hover Performance Test Results for Multicopter UAS Vehicles," 2016.

[21] M. Bernard, K. Kondak, I. Maza, and A. Ollero, "Autonomous transportation and deployment with aerial robots for search and rescue missions," J. F. Robot., vol. 28, no. 6, pp. 914-931, 2011.

[22] The Verge. "Drones could make Amazon's dream of free delivery profitable." [Online]. Retrieved from http://www.theverge.com/2015/6/3/8719659/amazon-prime-airdrone-delivery-profit-free-shipping-small-items, 16 February 2016.

[23] W. C. Young and R. G. Budynas, Roark's Formulas for Stress and Strain, vol. 7, no. 7th Edition. 2002.

[24] S. Driessens and P. E. I. Pounds, "Towards a more efficient quadrotor configuration," in 2013 IEEE/RSJ International Conference on Intelligent Robots and Systems, 2013, pp. 1386-1392.

\begin{tabular}{|c|c|c|c|c|c|c|c|c|c|c|c|c|c|c|c|}
\hline \multirow{2}{*}{$\begin{array}{l}\text { Multicopter } \\
\text { type }\end{array}$} & \multicolumn{15}{|c|}{ Number of segments 'ns' } \\
\hline & \multicolumn{15}{|c|}{ Apex angle ' $\beta$ ' } \\
\hline Quadcopter & 70,53 & & 101,57 & & 123,86 & & 126,92 & 129,20 & 130,65 & & 131,62 & 132,57 & 132,80 & & 133,17 \\
\hline Hexacopter & & 88,84 & & 116,02 & & 125,27 & & 129,20 & & 131,18 & & 132,57 & & 133,00 & \\
\hline
\end{tabular}

TABLE IV. EXAMPLE VALUES OF APEX ANGLES FOR DIFFERENT NUMBERS OF SEGMENTS. 